Please do not remove this page

RMIT

UNIVERSITY

\title{
Molecular dynamics study of polymer conformation as a function of concentration and solvent quality
}

Zhou, Zhongwu; Daivis, Peter

https://researchrepository.rmit.edu.au/esploro/outputs/9921864007101341/filesAndLinks?institution=61RMIT_INST\&index=null

Zhou, Z., \& Daivis, P. (2009). Molecular dynamics study of polymer conformation as a function of concentration and solvent quality. Journal of Chemical Physics, 130(22), 224904-1-224904-224910. https://doi.org/10.1063/1.3149858

Document Version: Published Version

Published Version: https://doi.org/10.1063/1.3149858

Repository homepage: https://researchrepository.rmit.edu.au

(C) 2009 American Institute of Physics.

Downloaded On 2023/04/26 19:55:24 +1000

Please do not remove this page 


\title{
Molecular dynamics study of polymer conformation as a function of concentration and solvent quality
}

\author{
Zhongwu Zhou and Peter J. Daivis ${ }^{\text {a) }}$ \\ School of Applied Sciences, RMIT University, G.P.O. Box 2476V, Melbourne, Victoria 3001, Australia
}

(Received 19 February 2009; accepted 14 May 2009; published online 10 June 2009)

\begin{abstract}
A coarse-grained model for molecular dynamics simulations of polymer solutions with variable solvent quality is proposed. This model allows solvent quality to be varied over the whole range from very poor to very good solvent conditions by varying a single parameter. The model has several advantages. All interactions are short ranged and repulsive, making the model very computationally efficient compared with other explicit solvent models that include the long-ranged attractive part of the interactions; the solvent is included explicitly, ensuring that the theta condition corresponds to a genuine cancellation of the solvent-mediated polymer-polymer interactions; and hydrodynamic interactions and entanglement can occur for all solvent conditions. The theta point is determined and the conformational properties of a 50-bead chain system are investigated over the whole concentration range from the dilute limit to the melt as a function of solvent quality. (C) 2009 American Institute of Physics. [DOI: 10.1063/1.3149858]
\end{abstract}

\section{INTRODUCTION}

Coarse-grained models of polymers such as the simple bead-spring and bead-rod models have been proven to be extremely useful for molecular dynamics (MD) simulations because they are computationally efficient compared with all-atom models and yet they still display the essential, universal physical features of polymer structure and dynamics. ${ }^{1,2}$ By using very short-ranged, hard-sphere-like interatomic interactions, computational efficiency can be maximized, enabling long chains to be studied over long simulation times. Computational studies of these simple model polymer

melts have been very successful in revealing the structural and rheological properties of linear, ${ }^{3}$ branched, ${ }^{4}$ and hyperbranched ${ }^{5}$ polymer melts at the molecular level, providing details of molecular behavior that are not readily accessible by experimental methods.

While there have been many MD simulation studies of polymer melts, there have been far fewer studies of polymer solutions using this technique. ${ }^{6,7}$ Brownian dynamics methods have been used extensively to study the structural and dynamic properties of dilute polymer solutions, but they become much more difficult to apply for concentrated solutions due to the difficulty of correctly accounting for hydrodynamic interactions at high polymer volume fractions. An important advantage of MD over the Brownian Dynamics method is that the exact hydrodynamic interactions emerge naturally from the motion of the explicitly included solvent molecules in MD, whereas they must be added using necessarily approximate and complicated mobility tensors in the Brownian dynamics method. ${ }^{8}$ Other techniques, such as the dissipative particle dynamics, smoothed particle hydrody-

\footnotetext{
${ }^{a)}$ Electronic mail: peter.daivis@rmit.edu.au.
}

namics, and Lattice Boltzmann simulation methods are also capable of including solvent effects explicitly, ${ }^{9}$ but they also discard some detail that is retained by MD.

Polymer-solvent interactions are of primary importance in determining the spatial configurations of the polymers in solution and hence they have significant impact on all conformation-dependent properties of polymer solutions. A reasonably complete description of the static and dynamic properties of polymer solutions has been provided by scaling and renormalization group theories, ${ }^{10,11}$ the concepts of static and hydrodynamic screening, the Rouse and Zimm theories of polymer dynamics, and the Doi-Edwards tube model for molecular motion in the presence of entanglements. ${ }^{12}$ Generally, the description provided by these theories applies best to very high molecular weight polymers in athermal solvents in the idealized dilute, semidilute and melt concentration regimes. Less attention seems to have been given to the transition from dilute to moderately concentrated solutions for low to moderate molecular weight polymers, where a true semidilute regime may not exist and a virial series description rather than the scaling description seems to apply. In this case, a complete account of the effect of polymer-solvent interactions on the chain conformation that includes both solvent quality and concentration effects remains elusive and further work is needed.

Several studies of static chain properties have been performed by Monte Carlo (MC) simulations with a coarsegrained bead-spring model for the polymer chains that accounts for solvent effects by introducing an effective polymer-polymer interaction. ${ }^{13-15}$ In the limit of a single chain in dilute solution ${ }^{14}$ a transition from swollen to collapsed coils can be observed by varying the temperature. In these studies, the change in concentration was replaced by the change of polymer density, and the thermodynamic effect of the solvent was implicitly represented by adjusting the effective interaction parameters among the beads of poly- 
mers, without explicitly introducing the solvent degrees of freedom. By making this approximation, one loses the distinction between concentration and density of a polymersolvent system as separate variables, and thus some important physics might be missed in some cases. ${ }^{16}$ For example, hydrodynamic interactions, which are vitally important for dynamic phenomena are completely absent in such a treatment.

Xiao and Heyes ${ }^{17}$ conducted Brownian dynamics simulations to examine the effect of the excluded volume (EV) interactions on the static and dynamical properties of model polymer solutions in the free-draining limit over a wide concentration range. They used a bead-spring model to describe the polymer chains at a coarse-grained level and a bead-bead pair potential to represent segment-segment interactions to characterize the EV effects. Their simulation results show that the coil size of polymers shrinks with increasing concentration in the dilute and concentrated regions but expands and increases again in the highly concentrated region. This disagrees with MC simulation results ${ }^{15}$ which show a continuous decrease in the polymer coil size in the case of a good solvent as the concentration increases from dilute to concentrated and further to highly concentrated solutions. Stoltz et al. ${ }^{18}$ recently studied the concentration dependence of the radius of gyration and the shear and extensional rheology of model $\lambda$-phage DNA in a good solvent using configurational bias $\mathrm{MC}$ for the static properties and the Brownian dynamics technique for the dynamic properties. They obtained good agreement between the results of simulations and predictions of scaling theory for the concentration dependence of the radius of gyration under good solvent conditions over a limited concentration range, from $c / c^{*}=1$ to $c / c^{*}=10$ for sufficiently long chains.

None of these above-mentioned approaches incorporate the solvent molecules explicitly. The hydrodynamic effect of the solvent is considered through a hydrodynamic interaction tensor plus a random force while the solvent-averaged potential of mean force between the polymer beads is represented by using adjustable pairwise interaction potentials between sites on a polymer chain.

From a physical point of view, the explicit incorporation of solvent molecules can have a dramatic impact on the polymer dynamics in solutions. A study of the collapse dynamics of single polymer chain in poor solvents has revealed a significant difference in the collapse behavior between simulations using Brownian dynamics methods and those using MD methods where the solvent was only explicitly included in the latter cases. ${ }^{6}$ Approximating the interactions between polymer beads by a solvent-mediated potential of mean force will also result in the absence of entanglement effects at the theta point because the effective potential will then be equal to zero.

There is clearly a need for a molecular simulation model that includes solvent quality effects and hydrodynamic interactions and still retains fundamental polymer properties such as the uncrossability of chains in concentrated solutions regardless of the solvent quality. Such a model should also be computationally tractable. The primary purpose of this paper is to propose and characterize a coarse-grained model for
$\mathrm{MD}$ and $\mathrm{MC}$ simulations that possesses these properties. Using this model, we then investigate the influence of solvent quality and polymer concentration on the conformation of polymer chains.

\section{METHODS}

\section{A. Molecular models}

The molecular model and simulation algorithm used in this work have been described previously. ${ }^{7}$ However, we summarize the main features here for completeness, and we will point out differences between the current work and our previous simulations as they arise.

Our molecular model treats all particles in the system, including solvent molecules, explicitly. The solvent molecules and the polymer beads are both modeled as spherical particles interacting though truncated and shifted LennardJones (LJ) potential energy functions. In our previous papers, the interaction parameters were all identical for polymerpolymer, solvent-solvent, and polymer-solvent interactions which resulted in good solvent conditions. In the current work, we allow these interaction parameters to differ so that the solvent quality can be varied. The polymer molecules consist of $N_{s}=50$ identical beads ("interaction sites"). Bond lengths between adjacent sites on the same polymer molecule are held constant by bond-constraint forces, determined by using Gauss' principle of least constraint. ${ }^{19}$

The potential energy function describing all nonbonded interactions (polymer-polymer, polymer-solvent, and solvent-solvent) is given by the truncated and shifted form of the LJ potential energy function,

$$
\phi(r)= \begin{cases}4 \epsilon_{\alpha \beta}\left[\left(\frac{\sigma_{\alpha \beta}}{r}\right)^{12}-\left(\frac{\sigma_{\alpha \beta}}{r}\right)^{6}\right]-\phi_{c}, & r \leq r_{c} \\ 0, & r>r_{c},\end{cases}
$$

where $r$ is the separation of two interaction sites, $\epsilon_{\alpha \beta}$ is the potential well depth for interactions between species $\alpha$ and species $\beta$, and $\sigma_{\alpha \beta}$ is the value of $r$ at which the unshifted potential is zero. Because we have two distinct types of interaction sites, $\alpha \beta$ could be $p p$, ss, or $p s$ for the polymerpolymer, solvent-solvent, and polymer-solvent interactions, respectively. The shift, $\phi_{c}$, which is equal to the value of the unshifted potential at the cutoff $r=r_{c}$, is introduced to eliminate the discontinuity in the potential energy. At distances greater than the cutoff distance $r_{c}$, the potential is zero. For this set of simulations, we have taken the cutoff point for the potential to be the position of the minimum in the LJ potential, $r_{c}=2^{1 / 6} \sigma$. In this case, $\phi_{c}=-\epsilon$ and the potential energy and force are both equal to zero at $r=r_{c}$. A LJ potential with this truncation point results in purely repulsive interactions, and it is often known as the WCA (Weeks Chandler Andersen) potential. ${ }^{20}$ This potential is convenient for computational work because it is short ranged, and therefore computationally undemanding, but still retains the essential physics, i.e., the repulsive (EV) interaction.

All interaction sites have equal mass $m_{i \alpha}=m$ and the bonds between polymer interaction sites have length $l=\sigma_{p p}$. This bond length is small enough to prevent chain crossings. 
The site-site WCA potential used in our simulations is purely repulsive, which obviously prevents two interaction sites from occupying the same space. At first glance, it would seem that this potential alone could not describe the complete range of solvent quality variation, i.e., from a poor solvent where the net attractive interactions between polymer segments lead to phase separation, to the theta point where the EV effect is absent, and further to a good solvent where the polymer segments repel each other and the EV interaction is strong. However, the EV interaction, and hence the solvent quality, is determined by a solvent-averaged potential of mean force, which results from the overall effect of the polymer-polymer and polymer-solvent interactions. ${ }^{21}$ When the solvent is explicitly included in the considerations, the effective polymer-polymer interaction depends not only on the direct polymer-polymer interactions though $\epsilon_{p p}$ but also on the polymer-solvent interactions through $\epsilon_{p s}$. In our simulations, instead of modifying the solvent quality by changing the temperature $T$, the very same effect is achieved by varying the interaction parameter, $\epsilon_{p s}$, between the polymer sites and solvent molecules. The change of $\epsilon_{p s}$ changes the strength of repulsive force between the polymer sites and solvent molecules so that the net effective EV interaction between polymer sites can be alternated through repulsive to neutral, and then to the attractive, which corresponds to the conditions of good, theta, and poor solvents, respectively. In our simulations, the other LJ parameters are set fixed to the values $\sigma_{p p}=\sigma_{p s}=\sigma_{s s}=1.0$ and $\epsilon_{p p}=\epsilon_{s s}=1.0$ and then $\epsilon_{p s}=1.0$ corresponds approximately (although not exactly, as we will see later) to an athermal condition. ${ }^{7}$ Increasing the value of this parameter corresponds to preferential repulsion of the solvent by a polymer bead relative to the polymerpolymer repulsion, decreasing the solvent quality. Therefore, in all simulations, the temperature can be kept at a constant value and only the parameter $\epsilon_{p s}$ is varied. This corresponds to a choice of solvents of different quality in an experimental context. In this study $\epsilon_{p s}$ ranges from 0.5 to 3.0.

\section{B. Simulation details}

The MD simulations were conducted in the NVT ensemble. The polymer site number fraction, $c_{p}$, is defined as the fraction of sites in the system belonging to polymer chains, and is used henceforward to describe the concentration of the system. In this paper we express all quantities in terms of polymer site (or equivalently, solvent molecule) reduced units for which the reduction parameters are the $\mathrm{LJ}$ interaction parameters $\epsilon_{p p}=\epsilon_{s s}, \sigma_{p p}=\sigma_{p s}=\sigma_{s s}$, and the mass $m$ of the polymer site. An overall reduced site density of $n^{\dagger}=n \sigma^{3}=0.84$ is used for all of our simulations with the polymer site fraction $c_{p}$ changing from 0 to 1 . The molecular center of mass kinetic temperature for both the polymer chains and solvent molecules is kept at $T^{\dagger}=k_{B} T / \epsilon_{s s}=1.0$ by using a thermostatting term derived from Gauss' principle of least constraint in the equations of motion. The mass of each site is $m_{p}^{\dagger}=m_{s}^{\dagger}=m_{s} / m=1.0$ and the reduced time is defined by $t^{\dagger}=\left(\epsilon_{s s} / m \sigma^{2}\right)^{1 / 2} t$. All quantities will be expressed in reduced units and the superscript dagger denoting reduced quantities will be omitted from here on.
The various polymer and solvent systems used in these simulations were generated from a melt of 50-site polymers consisting of a total of 12800 particles (sites). The model polymer solutions with different values of the concentration were obtained by breaking an appropriate number of polymer chains into single-site molecules to produce the desired number of solvent molecules in the system. Each of the produced systems was then equilibrated for 3000000 time steps before any production runs. Thereafter, typically three production runs of 3000000 time steps were conducted for different solvent quality conditions at each concentration. Conformational data quoted in Sec. III A were calculated by first averaging over 60 instantaneous trajectory files containing positions of all the polymer site positions in the simulation system and then averaging these over each of the three production runs.

The equations of motion of all particles are solved at each time step by a fourth-order Gear predictor-corrector scheme with a time step of 0.004 reduced time units. Bulk behavior is simulated via periodic boundary conditions (PBCs) and the minimum image convention. For polymer chains, the interaction between a given site and a periodic image of itself, or a nearby site on the same molecule is an unwanted and unphysical artifact of simulations that may occur due to the use of PBCs. In our simulations, the length of the cubical simulation box is about 24.8 reduced length units which is significantly larger than the maximum value of the rms radius of gyration, $\left\langle R_{g}^{2}\right\rangle^{1 / 2} \approx 4$.8. This ensures that no site on a given chain can interact directly with a correlated segment of any of the periodic images of the same chain. We also expect that long-ranged hydrodynamic interactions between periodic images of a given chain will be negligible in our simulations because even our lowest concentrations are high enough to provide sufficient hydrodynamic screening. To verify this, we also conducted simulations with larger simulation systems of total 43200 particles for the low concentration solutions. The results from the two different sized simulation systems were in agreement with each other, indicating that there were no detectable system-size effects in our results. This contrasts with the MD calculations of the self-diffusion coefficient of a single polymer chain in explicit solvent performed by Dunweg and Kremer $^{22}$ and Pierleoni and Ryckaert, ${ }^{23}$ in which it was necessary to correct for the influence of unscreened long-ranged hydrodynamic interactions.

\section{RESULTS AND DISCUSSION}

\section{A. Molecular dimensions}

In this section the influence of solvent quality on the molecular dimensions of model polymer chains over the entire concentration region is investigated. This study goes beyond most previous studies where the properties were investigated either in the athermal condition or in the vicinity of the theta point, usually without explicitly including the solvent molecules. In our work, the effect of solvent quality on the concentration dependence of the chain dimensions is examined under solvent quality conditions ranging from very 


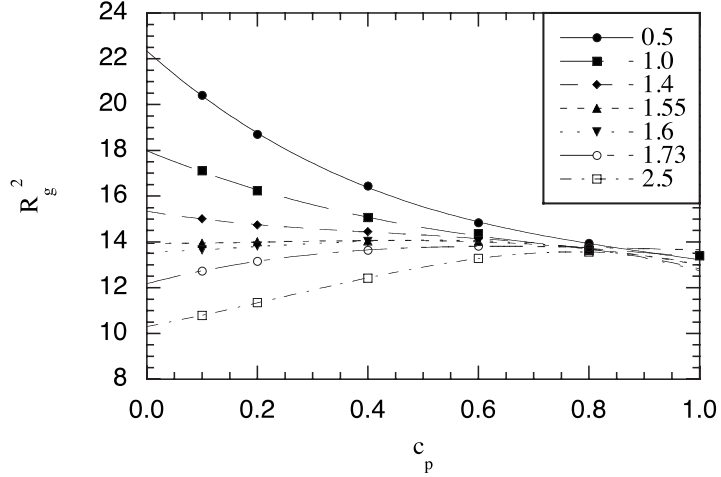

FIG. 1. Mean squared radius of gyration vs polymer concentration $c_{p}$ for different values of the polymer-solvent bead interaction strength, $\epsilon_{p s}$. Uncertainties are smaller than the plot symbols.

good to very poor by explicitly including the solvent molecules and continuously varying the polymer-solvent interaction parameter.

Polymer molecules in solution are typically coiled to occupy an elliptical region with a continually varying shape and orientation. ${ }^{24}$ The time average of the polymer conformation with respect to a set of axes with laboratory-fixed directions located at the center of mass of the molecule is a sphere with a dimension characterized by the radius of gyration, $R_{g}$, defined by

$$
R_{g}^{2}=\frac{1}{2 N_{s}^{2}} \sum_{i} \sum_{j}\left\langle r_{i j}^{2}\right\rangle,
$$

where $N_{s}$ is the number of sites in the chain and $r_{i j}$ is the magnitude of the distance between sites $i$ and $j$ on the molecule. This quantity depends on the quality of the solvent and polymer concentration.

Figure 1 shows the concentration dependence of the chain dimensions at different solvent qualities. For clarification we divide the curves in Fig. 1 into three groups. The first group includes three curves at $\epsilon_{p s}=0.5,1.0$, and 1.4 , representing good solvent conditions. The second includes two curves for $\epsilon_{p s}=1.55$ and 1.6, in the vicinity of the theta condition. The determination of the theta condition will be discussed in Sec. III B. The third group includes the curves for $\epsilon_{p s}=1.73$ and 2.5, representing poor solvent conditions. All of the data in Fig. 1 are very well fitted by cubic polynomial functions, as shown.

We can see that in good solvent conditions, the radius of gyration decreases with increasing concentration, indicating that the chain expansion induced by the solvent-mediated EV interaction gradually decreases. It should be noted that $\epsilon_{p s}=1.0$ might naively be expected to represent the athermal condition, as it does in lattice models of polymer solutions. ${ }^{21}$ This expectation would be exactly fulfilled for $N_{s}=1$, but due to the difference in partial specific volume between the pure solvent and the polymer in solution that exists because of the difference between the polymer bond length and average spacing of the solvent molecules, this will not be the case for chain molecules in this continuous space model. Decreasing $\epsilon_{p s}$ further results in even greater chain expansion, as shown by the results for $\epsilon_{p s}=0.5$ in Fig. 1. Precise determination of athermal conditions for this model would require detailed

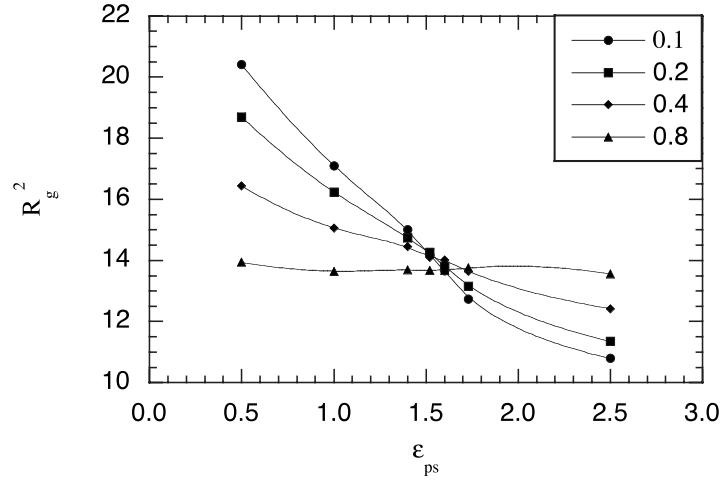

FIG. 2. Mean squared radius of gyration versus solvent quality for different values of the concentration, $c_{p}$. Uncertainties are smaller than the plot symbols.

analysis of the thermodynamics of the solutions. We can also see that in poor solvent conditions, the radius of gyration of the polymer increases with increasing concentration, displaying the opposite behavior to that exhibited by the polymer in good solvents.

In theta solvent conditions, the EV effect disappears as the polymer chains behave like ideal random walks and it might be expected that the radius of gyration should be approximately independent of concentration. While this is roughly true at low concentrations, our results show that this does not hold over the entire concentration range. We observe that for values of $\epsilon_{p s}$ at which the initial slope of the radius of gyration is approximately zero, it increases slightly at intermediate concentrations and then decreases when the concentration approaches 1.0. The maximum values of the radius of gyration occur at polymer concentrations of around 0.2 and 0.5 for the conditions of $\epsilon_{p s}=1.55$ and 1.6, respectively. This result suggests that even though there are no explicit three-body interatomic forces in this molecular model, three-body correlations definitely exist, and the third and higher order virial coefficients need not necessarily be equal to zero at the theta point. This is precisely what is needed to prevent the total collapse of polymer chains under very poor solvent conditions (see p. 116 of Ref. 21).

While the polymers exhibit different conformational structures when they are present in the solvents of different qualities, all the curves at the conditions of good, theta, and poor solvents coincide at very high concentration, greater than about $c_{p}=0.8$, as shown in Fig. 1, and the radius of gyration can be extrapolated to its pseudoideal dimension in the melt state as suggested by Flory's ideality hypothesis. ${ }^{21}$

Figure 2 displays the solvent quality dependence of the polymer mean squared radius of gyration at different polymer concentrations. It can be seen that the polymer size decreases as we increase the value of $\epsilon_{p s}$ at the concentrations of $0.1,0.2$, and 0.4 . This indicates that the polymer chains in the solutions shrink from a highly expanded shape to a random walk coil as the EV effect is gradually reduced by the gradually increased repulsive force between the polymer sites and solvent molecules. Once the EV effect is fully cancelled, a further increase of the repulsive force (corresponding to $\epsilon_{p s}>1.6$ ) between the polymer sites and solvent molecules causes contraction of the polymer molecules to 
TABLE I. Overlap concentration for different values of the solvent quality.

\begin{tabular}{llllllll}
\hline \hline$\epsilon_{p s}$ & 0.5 & 1.0 & 1.4 & 1.52 & 1.6 & 1.73 & 2.5 \\
$c_{p}^{*}$ & 0.17 & 0.22 & 0.25 & 0.26 & 0.27 & 0.29 & 0.35 \\
\hline \hline
\end{tabular}

dimensions which are smaller than the random coils. Furthermore, the contraction of polymer dimensions diminishes when the strength of repulsive forces between the polymer sites and solvent molecules is high enough. For example, the values of the mean squared radius of gyration at concentration of 0.1 are nearly the same, with values of 10.7 and 10.8 for $\epsilon_{p s}=2.5$ and $\epsilon_{p s}=3.0$ (not shown in Fig. 2), respectively.

Although the computed size of the polymer chains is no longer decreasing as $\epsilon_{p s}$ increases in this region, the polymers may still not reach a fully collapsed state in our simulations. Simulation studies ${ }^{6}$ on the collapse of single polymer chain have observed around $\sqrt{5}$ times reduction in the polymer radius of gyration from the athermal condition to the collapsed state. The computed maximum reduction in the radius of gyration in our simulations for a concentration of 0.1 is only close to $\sqrt{2}$. In contrast to the single chain system, multiple polymer chains in a very poor solvent have the opportunity to form aggregates or even phase separate instead of reaching the fully collapsed state. Direct visualization of configurations of our poorest solvent system at the lowest concentrations studied did, in fact, show the formation of aggregates and partial phase separation. These phase separating systems equilibrate very slowly. The results shown for $\epsilon_{p s}=2.5$ at low concentration are sometimes inconsistent with the trends shown by the other systems (see, for example, Fig. 4) and should therefore be treated with caution.

From Fig. 2 it can be seen that the impact of solvent quality on the polymer chain dimensions becomes less significant as the polymer concentration increases and is almost completely absent in highly concentrated solutions. For the highly concentrated solution of $c_{p}=0.8$ the mean squared radius of gyration is nearly constant with a value of $13.6 \pm 0.04$ at all values of $\epsilon_{p s}$ except for $\epsilon_{p s}=0.5$. For this very good solvent condition a value of $13.9 \pm 0.05$ for the mean squared radius of gyration is obtained. It is interesting to note that at the concentration of 0.8 , regardless of the solvent quality, the measured values of the squared radius of gyration are consistently higher than the value of $13.4 \pm 0.03$ obtained for the melt.

Various theories can be used to explain the solvent quality dependence of the radius of gyration in polymer solutions within the different concentration regimes. While theories of polymer conformation for the idealized infinitely dilute, semidilute and polymer melt regimes are very successful, more needs to be done for the concentrated region. According to de Gennes, ${ }^{10}$ polymer molecules begin to feel the presence of other molecules at the overlap concentration where the segment density inside the polymer coil becomes comparable to the overall segment density in the system. It is often assumed that the concentration dependence of the radius of gyration of long-chain polymers should be negligible below the overlap concentration $c_{p}^{*}$ and it should begin to vary due to the screening of the EV interactions in the semidilute concentration region. We assume that crossover occurs when the aver-

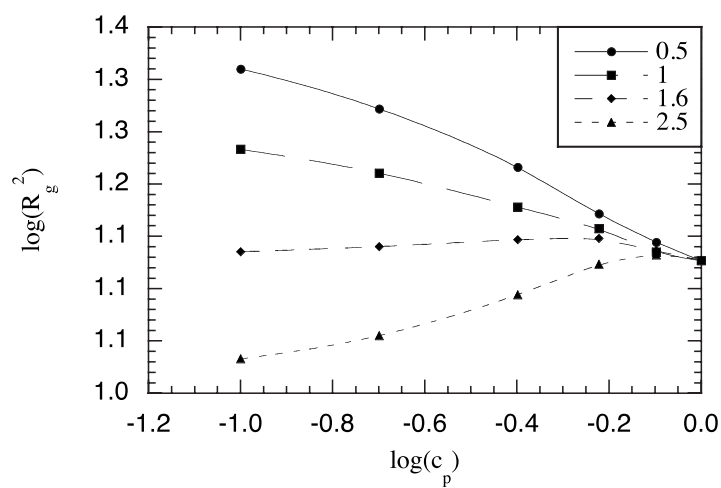

FIG. 3. Log-log plot of the squared radius of gyration against concentration for different values of the solvent quality from very poor (bottom) to very good (top). Statistical uncertainties are smaller than the plot symbols. The smooth curves are provided to guide the eye.

age concentration of the solution is equal to the concentration within the domain (assumed to be spherical) of the polymer molecule using the radius of gyration at the overlap concentration, which can therefore be calculated from

$$
c_{p}^{*}=\frac{N_{s}}{\frac{4}{3} \pi\left(R_{g}^{*}\right)^{3} \rho},
$$

where $R_{g}^{*}$ is the root mean square radius of gyration of the polymer chains at $c_{p}^{*}$ (which is quite close to the infinite dilution value). Note that the factor of number density $\rho$ arises from our use of polymer site number fraction rather than polymer site number density as our concentration unit. Since the $R_{g}^{*}$ data are not always available, an iterative method was used to determine the overlap concentration for each value of $\epsilon_{p s}$ and the results are listed in Table I. It should be remembered that the onset of polymer coil overlap does not necessarily result in a sudden or obvious change in solution properties. Rather it is usually observed to be a gradual change that may occur over a range of concentrations. Evidently the overlap concentration is dependent on the solvent quality for a particular kind of polymer. It is often assumed that the concentration dependence of the radius of gyration of long-chain polymers is negligible in the dilute region below the overlap concentration $c_{p}^{*}$, as the interchain interactions can be neglected. However, using the overlap concentration data given in Table I it can be seen from Fig. 1 that this is not the case for our systems except in the vicinity of theta condition.

Using the blob model and scaling theory, Daoud et al. ${ }^{25}$ obtained a power law relationship between macromolecular size and solution concentration in the semidilute regime given by

$$
R_{g}^{2} \propto c_{p}^{(2 \nu-1) /(1-3 \nu)},
$$

where the value of $\nu$ for an athermal solution is equal to 0.588 when calculated from renormalization group theory or 0.6 according to mean field theory. ${ }^{21}$ These values result in values of -0.23 and -0.25 , respectively, for the exponent of $c_{p}$ in Eq. (4). To test the validity of this relationship for our data, we plotted $\log \left(R_{g}^{2}\right)$ against $\log c_{p}$ in Fig. 3 and the effective exponents calculated from $d \log R_{g}^{2} / d \log c_{p}$ in Fig. 4 for four different values of the solvent quality. 


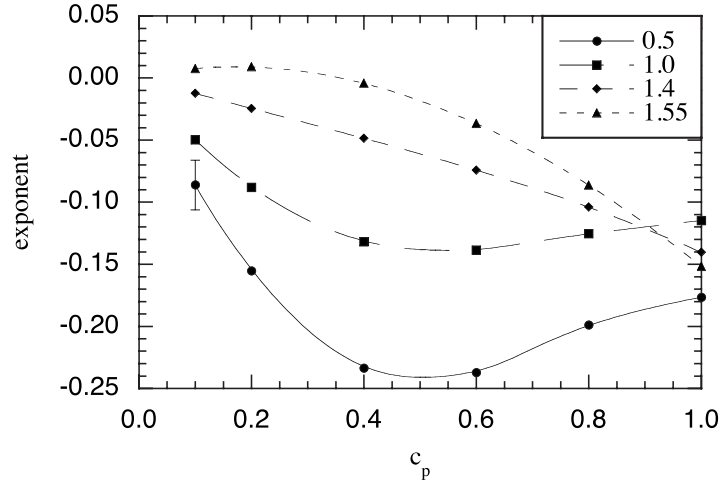

FIG. 4. Effective concentration scaling exponent for the squared radius of gyration for solvent qualities ranging from a very good solvent (bottom) to the theta state (top). The smooth curves through the data serve only as a guide for the eye. The error bar shown is typical for the whole data set.

The data in Fig. 4 show that the exponent for the two good solvents $\left(\epsilon_{p s}=0.5\right.$ and $\left.\epsilon_{p s}=1.0\right)$ decreases until the concentration reaches 0.4 and then remains nearly constant as the concentration is increased to 0.8. Experimental results for long-chain polymer solutions show that the absolute value of the slope of $\log \left(R_{g}^{2}\right)$ versus $\log c_{p}$ increases in magnitude with polymer concentration and exceeds 0.25 when the solution becomes concentrated. ${ }^{26}$

The MC simulation results of Olaj et al. ${ }^{15}$ for systems of 50-bead polymers (which do not explicitly include the solvent molecules) showed that the value of the exponent in Eq. (4) decreases with increasing concentration in the low concentration region and are scattered around the predicted value when the polymer density is larger than the crossover density. ${ }^{15}$ However, their results did not extend to sufficiently high densities to enable a direct comparison with our high concentration results. This becomes clear when it is observed that their results did not satisfy Flory's ideality hypothesis, i.e., that the radius of gyration of the polymer in a good solvent should be equal to the value for the theta solvent at sufficiently high concentrations. The general shape of the plots for our two good solvent systems is similar to the experimental results and MC simulation results, and the value of the exponent for the very good solvent condition $\left(\epsilon_{p s}=0.5\right)$ in the concentration region between 0.4 and 0.8 is about $-0.23 \pm 0.02$ which agrees well with the predicted value. The value of the exponent is about $-0.14 \pm 0.02$ for $\epsilon_{p s}=1.0$, which indicates that the athermal state may correspond to a value of $\epsilon_{p s}<1.0$ rather than $\epsilon_{p s}=1.0$ as was assumed in our previous work. ${ }^{7}$ This is supported by our analysis of the internal chain statistics shown later. Our previous MD simulations on shorter chain systems of 20-site polymers with $\epsilon_{p s}=1.0$ gave a magnitude of the exponent that was even lower than that found here, ranging from approximately -0.05 to -0.1 . Taken together, these results indicate that the scaling law prediction should be observable with our molecular model as the chain length is increased and the precise location of the athermal condition is identified.

\section{B. Theta state}

The $\theta$-state for a polymer solution can be defined as the state in which an isolated chain in solution behaves as ran-
TABLE II. Results of fits to Eq. (5)

\begin{tabular}{lcrrr}
\hline \hline$\epsilon_{p s}$ & $R_{g}^{2}(0)$ & $b_{1}$ & $b_{2}$ & \multicolumn{1}{c}{$b_{3}$} \\
\hline 0.5 & 22.34 & -0.9602 & 0.8521 & -0.3002 \\
1.0 & 18.26 & -0.7201 & 0.9095 & -0.5059 \\
1.4 & 15.34 & -0.2565 & 0.3909 & -0.2983 \\
1.5 & 14.53 & -0.0360 & -0.0355 & -0.0306 \\
1.52 & 14.29 & -0.0632 & 0.2030 & 0.2584 \\
1.55 & 13.93 & 0.0028 & 0.1335 & -0.2045 \\
1.6 & 13.53 & 0.0853 & 0.1297 & -0.2730 \\
1.73 & 12.18 & 0.5162 & -0.6309 & 0.2358 \\
2.5 & 10.30 & 0.4399 & 0.4378 & -0.6169 \\
\hline \hline
\end{tabular}

dom coil, or when the second virial coefficient vanishes. ${ }^{27} \mathrm{~A}$ common approach for varying the solvent quality in both simulations and experiments is to change the temperature directly. If simulations are conducted on polymers with different numbers of sites or beads $N$ at various temperatures $T$, the $\theta$-temperature can be determined as the temperature at which curves for $R_{g}^{2} / N$ versus $T$ for different $N$ intersect. ${ }^{14}$ Instead of using this approach, we vary the solvent quality in this study by modifying the interactions between the polymer sites and solvent molecules by varying the polymer-solvent interaction parameter $\epsilon_{p s}$. As shown in Sec. III A this allows us to cover the whole solvent quality range from a very good solvent through the theta point to a very poor solvent. In this study, we focus on the concentration dependence of the conformational properties of the polymers rather than the molecular weight dependence. Since we only have data for one chain length, we cannot use the chain length dependence of the radius of gyration to determine the theta point of our solutions. Instead, it is more convenient to make use of the fact that at sufficiently low concentrations, the concentration dependence of the radius of gyration is proportional to the second virial coefficient of the solution. Therefore, the initial slope of a plot of radius of gyration versus concentration should be zero at the theta point.

To show this more clearly, we express the squared radius of gyration as a power series in the concentration,

$$
R_{g}^{2}(c)=R_{g}^{2}(0)\left(1+b_{1} c+b_{2} c^{2}+b_{3} c^{3} \cdots\right),
$$

where $R_{g}^{2}(0)$ is the squared radius of gyration at infinite dilution and the coefficients $b_{1}, b_{2}, b_{3}$, etc., depend on the solvent quality. Theoretical expressions for the expansion coefficients were derived by Yamakawa [see section 27b(ii) of Ref. 27]. For our current purposes, it is sufficient to make the point that all of the theoretical approaches find that the coefficient $b_{1}$ is directly proportional to $-\beta$, the negative of the binary cluster integral, and hence also the negative of the second virial coefficient. This suggests that the coefficient $b_{1}$ in Eq. (5) should have a negative value in good solvent systems, a positive value in poor solvent systems, and a value of zero in theta solvent systems. Using a third order polynomial to fit Eq. (5) to the data presented in Fig. 1, values of $R_{g}^{2}(0)$, $b_{1}, b_{2}$, and $b_{3}$ were obtained, as shown in Table II.

Figure 5 shows the relationship between the linear term coefficient $b_{1}$ and polymer-solvent interaction parameter $\epsilon_{p s}$. The results shown in Fig. 5 do not include the data for $\epsilon_{p s}=2.5$, which appears to deviate from the trend of the other 


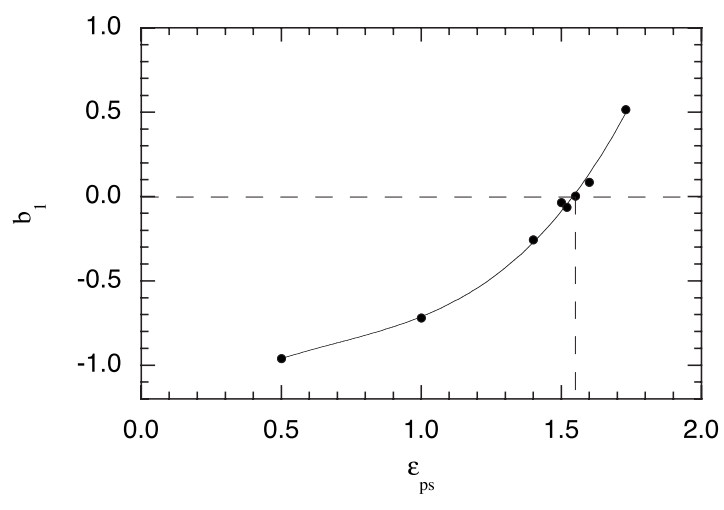

FIG. 5. Coefficient of the linear term in Eq. (5) vs solvent quality.

data. This deviation can be attributed to the formation of aggregates or partial phase segregation at this very poor solvent quality. As predicted, the value of $b_{1}$ increases from negative to positive as the solvent quality changes from good to poor, and the theta point is therefore determined as the value of $\epsilon_{p s}=1.55$.

Alternatively, one may use the ratio of mean squared end to end distance $R^{2}$ to the mean squared radius of gyration $R_{g}^{2}$ as a criterion to differentiate between good and poor solvent regimes and define the theta point. According to theory, a ratio $R^{2} / R_{g}^{2}$ of 6 is expected for sufficiently long linear Gaussian chains (i.e., at the theta point). A higher ratio is expected for a long polymer in a good solvent and a lower ratio is expected for a polymer in poor solvent. Such an approach is convenient in molecular simulations because both $R^{2}$ and $R_{g}^{2}$ are easily accessible quantities. The definition of the theta state by this method may not exactly correspond to the point at which the second osmotic virial coefficient is equal to zero, but it may serve as a convenient and reasonably accurate approximation to it. ${ }^{28}$ The results for the ratio $R^{2} / R_{g}^{2}$ against polymer concentration for three particular solvent quality conditions, the athermal, theta, and poor conditions, are depicted in Fig. 6. The values of the ratio in the limit of zero concentration obtained from a second order polynomial fit for all solvent quality conditions examined are shown in Table III.

The results in Table III indicate that the ratio is within uncertainties of the expected value of 6 for the melt, and at infinite dilution this value is greater for good solvent condi-

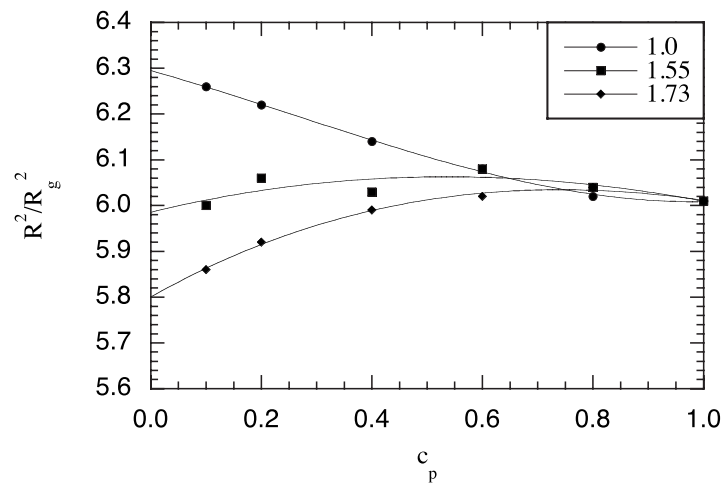

FIG. 6. Ratio of mean squared end to end distance to mean squared radius of gyration vs concentration for different values of the solvent quality.
TABLE III. Values of $R^{2} / R_{g}^{2}$ for various solvent quality conditions at the zero concentration limit and for the melt. Uncertainties are less than 0.04 .

\begin{tabular}{lllllllllll}
\hline \hline$\epsilon_{p s}$ & 0.5 & 1.0 & 1.4 & 1.5 & 1.52 & 1.55 & 1.6 & 1.73 & 2.5 & Melt \\
$R^{2} / R_{g}^{2}$ & 6.60 & 6.29 & 6.12 & 6.11 & 6.10 & 5.99 & 6.01 & 5.80 & 5.27 & 6.01 \\
\hline
\end{tabular}

tions, but lower for poor solvent conditions, as expected. The value of the ratio in the zero concentration limit at $\epsilon_{p s}=1.0$ is 6.29 , in good agreement with the value of 6.302 calculated for a polymer in an athermal solvent by Jannink and des Cloizeaux. ${ }^{29}$ The value obtained for $\epsilon_{p s}=0.5$ is even larger, at 6.60. This larger than expected value may indicate that the value of $\epsilon_{p s}=0.5$ corresponds to a stronger than athermal solvent, or more likely that the swelling is enhanced for short chains due to the nonuniform nature of the chain expansion, which is greater at the center of the chain. ${ }^{29}$ This is consistent with the fact that we obtained a value of 6.60 for $\epsilon_{p s}=1.0$ with a chain length of 20 in our previous work. ${ }^{7}$ Studies of longer chains would conclusively resolve this issue.

In the vicinity of theta point ( $\epsilon_{p s}$ between 1.55 and 1.60), the value obtained for $R^{2} / R_{g}^{2}$ is around 6 as predicted by theory for linear Gaussian chains. When the data listed in Table III are plotted in Fig. 7, the curve crosses over 6 at $\epsilon_{p s}$ nearly equal to 1.55 . This value matches the theta point as determined above from the condition that the initial slope of the radius of gyration versus concentration should be zero at the theta point. It can be seen from Fig. 6 that the three curves at different solvent conditions converge to the value for the melt when the polymer concentration reaches a very high value, around 0.8 (this is also the case with all other solvent quality conditions which are not shown in the figure). The range of values of $R^{2} / R_{g}^{2}$ at the concentration of 0.8 for all values of $\epsilon_{p s}$ examined is between 6.01 and 6.04, compared with 6.01 for the melt. It is interesting to observe that the value of $R^{2} / R_{g}^{2}$ is equal to 6.0 at the theta condition for both concentration limits, infinite dilution and as the melt is approached, but in semidilute and concentrated solutions the ratio is slightly larger than the theoretical value for a Gaussian chain.

If we compare Fig. 6 and Fig. 1, we can see that both $R_{g}^{2}$ and the ratio $R^{2} / R_{g}^{2}$ display a similar trend as the concentration increases for different solvent quality conditions. This trend was observed previously by Olaj et al. ${ }^{15}$ who proposed

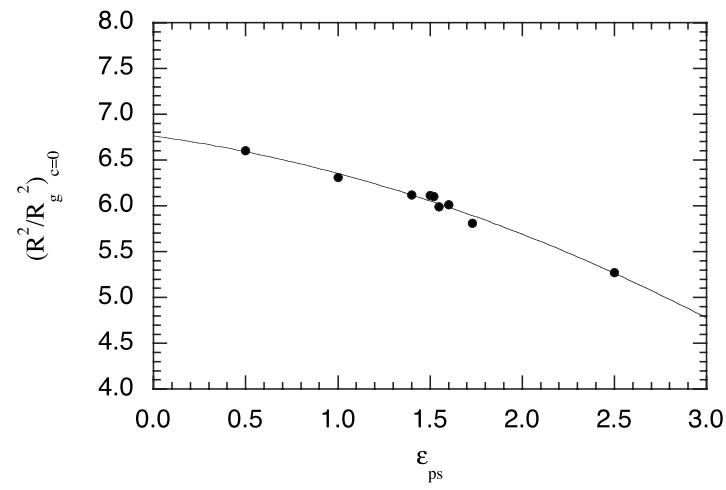

FIG. 7. Mean squared end to end distance divided by mean squared radius of gyration in the zero concentration limit vs solvent quality. 


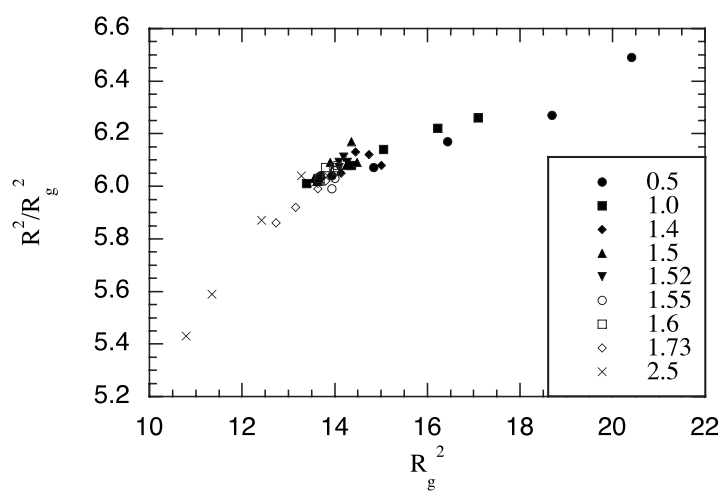

FIG. 8. Squared end to end distance divided by squared radius of gyration, plotted against radius of gyration, showing that the data approximately fall on a master curve regardless of solvent quality and concentration.

that a plot of $R^{2} / R_{g}^{2}$ versus $R_{g}^{2}$ should give a master curve for all different concentrations and different solvent quality conditions. We have plotted our data in the same way in Fig. 8, which shows that a reasonably good reduction to a master curve is obtained for our data. While Olaj et al. ${ }^{15}$ found that a master curve was reasonably well followed when they plotted data from lattice MC simulations of a single polymer chain in a surrounding matrix with varying solvent quality and concentration, their off-lattice MC simulations resulted in two distinct curves, one for the good solvent data and the other for the theta systems. A possible explanation for this is that their use of qualitatively different potential energy functions for the theta solvent and good solvent simulations has given rise to an inconsistency between the two data sets. This might be caused, for example, by different prefactors in the relationship between the radius of gyration and the chain length for different solvent conditions. This does not appear to be the case in our results, although more precise data in the region near $R^{2} / R_{g}^{2}=6.0$ might show discrepancies.

\section{Chain statistics}

It is also of interest to analyze the site statistics for our simulation results. In the limit of infinite dilution the mean squared distance $R_{i j}^{2}$, between any two sites $i$ and $j$ on the same chain can be described by the blob model. For a theta solvent or an athermal solvent, we expect the mean squared distance between two sites to be described by a power law

$$
R_{i j}^{2}=\left\langle\left(\mathbf{r}_{j}-\mathbf{r}_{i}\right)^{2}\right\rangle \propto|j-i|^{2 v},
$$

where $\nu$ is an exponent that depends on the solvent quality, with $\nu=0.6$ for a polymer in a good solvent, $\nu=0.5$ for a theta solvent or polymer melt, and $\nu=1 / 3$ for a very poor solvent. $^{21}$

The effect of $\epsilon_{p s}$ on the scaling behavior is depicted in Fig. 9 as plots of $\log R_{i j}^{2}$ versus $\log |j-i|$ for different solvent quality conditions at the polymer concentration of 0.1 . Straight lines indicate the lines of best fit over the range $|j-i|>30$, and the value of the gradient, $\nu$, decreases gradually as $\epsilon_{p s}$ increases. For values of $|j-i|<30$, deviations from linearity were observed in the log-log plots, indicating that for $N=50$, the data are well described by a power law only within a fairly narrow range of monomer separations,

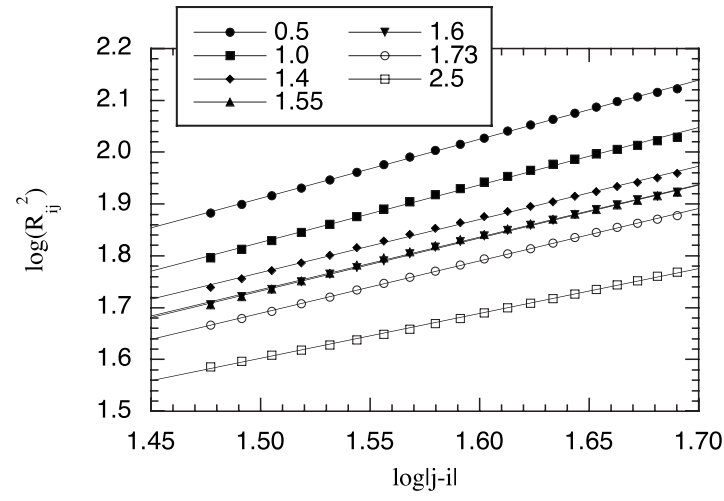

FIG. 9. Internal chain site statistics at polymer concentration $c_{p}=0.1$. The data are well described by a power law relationship for all solvent qualities for monomer separations in the range $|j-i|>30$.

$30<|j-i|<50$. Therefore, the exponents obtained in this way should be regarded as effective exponents. Studies of much longer chains would be needed in order to determine the asymptotic values of the exponents, if such values exist.

We have analyzed all $R_{i j}^{2}$ versus $|j-i|$ data in this way to obtain values of $\nu$ for various solvent quality conditions at different polymer concentrations and the results are shown in Fig. 10. It can be seen from Fig. 10 that most values of $\nu$ range between 0.5 and 0.53 , except for a few cases of low concentration solutions under very good or very poor solvent conditions. For the polymer melt we obtained a value of 0.514 , somewhat larger than the expected value of 0.5 . After obtaining the exponents for each solvent quality and concentration from plots similar to Fig. 9 and extrapolating the data shown in Fig. 10 to $c_{p}=0$, the values of the exponent $\nu$ in the limit of zero concentration are obtained and listed in Table IV.

As shown in Table IV and Fig. 10, the values of $\nu$ at either the vicinity of the theta point or at high concentrations for good solvent conditions are approximately equal to that of the melt. This suggests that the chain statistics for the theta solvents or higher concentrations tend toward random walk behavior as predicted by Flory. However, they are consistently slightly higher than the theoretical value of 0.5 predicted by the random walk model. Wittmer et al. ${ }^{30,31}$ recently discussed deviations from Flory's ideality hypothesis. Using

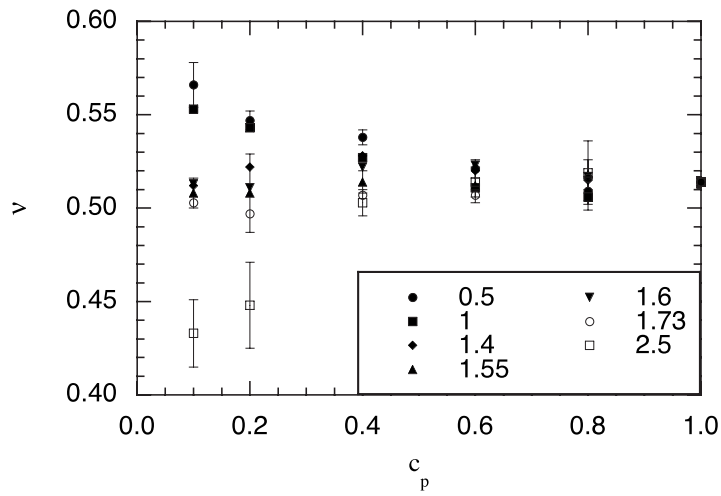

FIG. 10. Concentration dependence of the exponents for internal monomer separations at different values of the solvent quality. Error bars are shown for three representative values of solvent quality. 
TABLE IV. Values of power law exponent $\nu$ for various solvent quality conditions in the zero concentration limit and for the melt. Uncertainties are less than 0.01 .

\begin{tabular}{|c|c|c|c|c|c|c|c|c|c|c|}
\hline$\epsilon_{p s}$ & 0.5 & 1.0 & 1.4 & 1.5 & 1.52 & 1.55 & 1.6 & 1.73 & 2.5 & Melt \\
\hline$\nu$ & 0.574 & 0.565 & 0.506 & 0.512 & 0.527 & 0.507 & 0.504 & 0.501 & 0.397 & 0.514 \\
\hline
\end{tabular}

numerical data obtained from MC simulations with the bond fluctuation model and MD simulations with the bead-spring model, they were able to show that algebraically decaying residual correlations exist in the bond-bond correlation function for a polymer melt. The simulation results were consistent with their theoretical predictions. This means that the random walk model is only obeyed approximately, and the effective Flory exponent is expected to be slightly larger than 0.5 , even for very large chain lengths.

For the good solvents $\left(\epsilon_{p s}=1.0,0.5\right)$ the value obtained for the exponent in the dilute limit is around 0.57 which is smaller than the theoretical value of 0.588 while the value of 0.397 in the very poor solvent $\left(\epsilon_{p s}=2.5\right)$ is higher than the expected value of $1 / 3$. The discrepancy may be due to the uncertainty caused by the extrapolation because the concentrations examined may not be low enough. It is also possible that the ideal dilute athermal and very poor solvent conditions may not actually be achieved. This is definitely the case for the very poor solvent conditions as we direct visualization of configurations showed that for polymer solutions of multiple chains the aggregation of polymers and partial phase separation occurs instead of the total collapse to tightly spherical coils when the solvent quality gets worse. However, for good solvent conditions this is a more subtle problem. When the interaction between the polymer sites and solvent molecules are explicitly considered, as we have done in this study, we have the option of setting $\epsilon_{p s}=0$. This is a trivial but nonphysical way of achieving the athermal condition that has been previously used in the simulations in which the solvent was not explicitly included. Then the concentration must be varied by changing the polymer density instead of the concentration. Any nonzero value of $\epsilon_{p s}$ and nonzero $\sigma_{p s}$ will not be equivalent to this version of the athermal condition. On the other hand, it should also be possible to achieve the athermal condition by adjusting the polymer-solvent interaction parameter so as to achieve a value for the Flory interaction parameter $\chi$ of exactly 0.5 . This will require a careful investigation of the thermodynamics in addition to the study of conformational data that we have carried out here.

We have observed that the gradient values are not exactly constant when plotting $\log R_{i j}^{2}$ versus $\log |j-i|$ for the whole $|j-i|$ range. The fits shown in Fig. 9 were deliberately restricted to the range of $\log |j-i|$ over which a straight line was obtained, i.e., $|j-i|>30$ with a maximum value of 49 for a 50-site chain, for the purpose of analyzing the extrapolation of the results to zero concentration. It is not surprising that the gradients obtained for smaller values of $|j-i|$ are slightly higher than for larger values of $|j-i|$ because of the residual short range steric effect associated with the local chain stiffness of our relatively flexible polymer model. Likewise, the slopes at high values of $|j-i|$ could be affected by contacts with other chains-i.e., the beginning of screen- ing effects. These features are expected to become much more distinct when long chains are studied and a true semidilute region with strong overlap but low polymer volume fraction is possible. Full consistency with the thermal blob model should therefore not be expected due to the relatively short length of the chains we have studied here and in our previous work. ${ }^{7}$

\section{CONCLUSIONS}

We have developed a simple, computationally efficient model of polymer solutions for MD simulations that allows the solvent quality to be varied over the whole range from a very poor solvent where chain collapse and phase separation occur, to very good solvent conditions, where the chains are expanded and the solutions are close to the athermal condition. The model only involves short-ranged repulsive interactions for the polymer-polymer, the solvent-solvent, and the polymer-solvent bead interactions. This model has the advantage that the theta condition is achieved by the genuine cancellation of the competing effects of polymer-polymer and polymer-solvent repulsion, with the result that corrections due to three-body correlations are still present at the theta point, despite the vanishing of the two-body EV. This fact that the direct polymer-polymer interaction is not zero at the theta point also means that entanglements can still occur even though the solvent-averaged effective pair interaction between polymer molecules is zero. This differs fundamentally from models that neglect the solvent and simply set the polymer bead interactions to zero. The presence of explicit solvent also has the advantage that the full hydrodynamic interactions between polymer beads emerge naturally.

The theta point was determined by finding the value of the polymer-solvent interaction parameter $\epsilon_{p s}$ at which the initial slope of the plot of radius of gyration versus concentration was zero. The value obtained was in good agreement with the value estimated by finding the value of $\epsilon_{p s}$ where the ratio $R^{2} / R_{g}^{2}$ was equal to 6.0 in the limit of zero concentration.

We also found that determination of athermal conditions is subtle. If the polymer and solvent had identical partial specific volumes as they are assumed to have in lattice models of polymer thermodynamics, the athermal condition would be expected to correspond to $\epsilon_{p s}=\epsilon_{p p}=\epsilon_{s s}$. However, this is not the case for the polymer bond length that we use here. This means that a more careful investigation of the thermodynamics of these solutions is required to determine the exact values of the interactions parameters that result in athermal solvent conditions. 


\section{ACKNOWLEDGMENTS}

This work was supported by the Victorian Partnership for Advanced Computing, through their eResearch Grants program.

${ }^{1}$ M. Kröger, Phys. Rep. 390, 453 (2004).

${ }^{2}$ P. Daivis, M. Matin, and B. Todd, J. Non-Newtonian Fluid Mech. 147, 35 (2007).

${ }^{3}$ P. Daivis, M. Matin, and B. Todd, J. Non-Newtonian Fluid Mech. 111, 1 (2003).

${ }^{4}$ J. Bosko, B. Todd, and R. Sadus, J. Chem. Phys. 124, 044910 (2006).

${ }^{5}$ T. Le, B. Todd, P. Daivis, and A. Uhlherr, J. Chem. Phys. 130, 074901 (2009).

${ }^{6}$ R. Chang and A. Yethiraj, J. Chem. Phys. 114, 7688 (2001).

${ }^{7}$ T. Kairn, P. Daivis, M. Matin, and I. Snook, Polymer 45, 2453 (2004).

${ }^{8}$ R. Jendrejack, D. Schwartz, J. de Pablo, and M. Graham, J. Chem. Phys. 120, 2513 (2004)

${ }^{9}$ P. Espanol, Lect. Notes Phys. 640, 69 (2004).

${ }^{10}$ P.-G. de Gennes, Scaling Concepts in Polymer Physics (Cornell University Press, Ithaca, 1979).

${ }^{11}$ Y. Oono, Adv. Chem. Phys. 61, 301 (1985).

${ }^{12}$ M. Doi and S. F. Edwards, The Theory of Polymer Dynamics (Clarendon, Oxford, 1986)

${ }^{13}$ I. Gerroff, A. Milchev, K. Binder, and W. Paul, J. Chem. Phys. 98, 6526 (1993)
${ }^{14}$ A. Milchev, W. Paul, and K. Binder, J. Chem. Phys. 99, 4786 (1993).

${ }^{15}$ O. Olaj, T. Petrik, and G. Zifferer, J. Chem. Phys. 107, 10214 (1997).

${ }^{16}$ K. Binder and A. Milchev, J. Comput.-Aided Mater. Des. 9, 33 (2002).

${ }^{17}$ C. Xiao and D. M. Heyes, Phys. Rev. E 60, 5757 (1999).

${ }^{18}$ C. Stoltz, J. de Pablo, and M. Graham, J. Rheol. 50, 137 (2006).

${ }^{19}$ D. Evans and G. Morriss, Statistical Mechanics of Nonequilibrium Liquids (Academic, London, 1990).

${ }^{20}$ J. D. Weeks, D. Chander, and H. C. Andersen, J. Chem. Phys. 54, 5237 (1971).

${ }^{21}$ M. Rubinstein and R. Colby, Polymer Physics (Oxford University Press, Oxford, 2003)

${ }^{22}$ B. Dünweg and K. Kremer, Phys. Rev. Lett. 66, 2996 (1991).

${ }^{23}$ C. Pierleoni and J.-P. Ryckaert, J. Chem. Phys. 96, 8539 (1992).

${ }^{24}$ K. Solc and W. Stockmayer, J. Chem. Phys. 54, 2756 (1971).

${ }^{25}$ M. Daoud, J. Cotton, B. Farnoux, G. Jannink, H. Sarma, G. Benoit, C. Duplessix, C. Picot, and P. de Gennes, Macromolecules 8, 804 (1975).

${ }^{26}$ K. Adachi, Y. Imanishi, T. Shinkado, and T. Kotaka, Macromolecules 22, 2391 (1989)

${ }^{27}$ H. Yamakawa, Modern Theory of Polymer Solutions (Harper \& Row, New York, 1971)

${ }^{28}$ M. Lisal and I. Nezbeda, J. Chem. Phys. 119, 4026 (2003).

${ }^{29}$ G. Jannink and J. des Cloizeaux, J. Phys.: Condens. Matter 2, 1 (1990).

${ }^{30}$ J. Wittmer, P. Beckrich, A. Johner, A. N. Semenov, S. P. Obukhov, H. Meyer, and J. Baschnagel, EPL 77, 56003 (2007).

${ }^{31}$ J. Wittmer, P. Beckrich, F. Crevel, C. C. Huang, A. Cavallo, T. Kreer, and H. Meyer, Comput. Phys. Commun. 177, 146 (2007). 